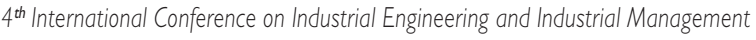

XIV Congreso de Ingeniería de Organización

Donostia - San Sebastián, September $8^{\text {th }}-10^{\text {th }} 2010$

\title{
Desencadenantes, resultados y factores críticos de éxito en los sistemas de participación del personal. Resultados de un estudio en España
}

\author{
Triggers, results and key factors in Employee Participation Systems. \\ Results of a study in Spain
}

\author{
J. Carlos Prado Prado, Jesús García Arca, Ana Mejías Sacaluga y Arturo J. Fernández González \\ Grupo de Ingeniería de Organización (GIO). Dpto. de Organización de Empresas y Marketing. \\ Escuela Técnica Superior de Ingeniería Industrial de Vigo. \\ Universidad de Vigo. Campus Lagoas-Marcosende, c/ Maxwell, 363 I 0.Vigo. \\ jcprado@uvigo.es jgarca@uvigo.es mejias@uvigo.es ajfdez@uvigo.es
}

Fecha de recepción: 8-9-2010

Fecha de aceptación: 27-10-2010

Resumen: Muchas empresas deciden implantar planes de mejora continua, cuyo éxito está fundamentado en la participación de toda la organización, la cual puede estructurarse en Sistemas de Participación del Personal (SPS). Esta ponencia tiene tres objetivos; por un lado, identificar y valorar los principales motivos para implantar SPs; por otro, identificar y valorar la importancia y dificultades asociadas a los factores de éxito mencionados en la literatura. Finalmente, identificar y valorar los principales resultados que las empresas logran con los SPs. Para ello, los autores han desarrollado un estudio en 40 empresas españolas con SPs.

Palabras clave: participación, competitividad, mejora continua.

Abstract: Many companies decide to implement continuous improvement plans, whose success is based on the participation of the entire organization, which can be structured by means of Employee Participation Systems (EPs). This paper has three objectives: on the one hand, to identify and assess the main reasons for implementing EPs; secondly, to identify and assess the importance and difficulties associated with the key factors mentioned in the literature. Finally, to identify and assess main results achieved with EPs. To do this, the authors have developed a study on 40 Spanish companies with EPs.

Keywords: participation, competitiveness, continuous improvement.

\section{Introducción}

En un mundo globalizado, con unos mercados turbulentos y volátiles caracterizados por unos altos niveles de competencia (Bolwijn y Kumpe, 1998; García y Prado, 2002) que afectan a todos los sectores, independientemente de su carácter industrial o de servicios, las empresas deben emprender acciones para, no sólo mantener sino, deseablemente, mejorar su posición competitiva. Esta búsqueda de mejoras en los procesos desarrollados por las empresas puede basarse, tanto en el ámbito de la inversión (en tecnologías, equipos e instalaciones) como en pequeñas mejoras que incrementen, gradualmente, su rendimiento.
Relacionado con este último aspecto, esto es, la mejora que casi no requiere de inversión, aparece la filosofía denominada «mejora continua» («continuous improvement») o «Kaizen», que constituye la base de diferentes enfoques, tales como Just in Time (IIT), Lean Production, Total Quality Management (TQM), 6 sigma, etc. En este contexto, Boer et al. (2000) definen la mejora continua como «un proceso organizado y planificado de cambio en toda la organización, orientado hacia la mejora de los resultados empresariales». Aunque tradicionalmente la mejora continua se ha enfrentado al concepto de innovación, ambos conceptos, lejos de ser considerados como excluyentes, deberían ser considerados como complementarios (Tonnessen, 2000; Boer, 200 I; García y Prado, 2002). 
Detrás de la implantación de un programa de mejora continua se encuentra la necesidad que tienen las empresas de incrementar su competitividad o rendimiento (Terziovski y Sohal, 2000). Esta mejora de la productividad o del rendimiento podría alcanzarse a partir de la mejora de la productividad, la mejora de la calidad, la reducción del tiempo de producción, la reducción de los costes o, de forma más genérica, la orientación hacia una mayor satisfacción del cliente (Boer et al., 2000; Grütter et al., 2002; Rapp y Eklund, 2002; Modarress et al., 2005). Aunque existe cierto consenso general en el mundo empresarial y académico en cuanto a que la aplicación de la mejora continua supone una mejora del rendimiento empresarial (Boer et al., 2000; Grütter et al., 2002), son pocos los trabajos que analizan esta relación de forma cuantitativa; entre ellos destaca el trabajo de Jorgensen et al. (2006).

En este sentido, las empresas que han aplicado satisfactoriamente la mejora continua presentan dos características importantes: en primer lugar, que han seguido un proceso «estructurado» de varias etapas que les han permitido lograr, progresivamente, compromiso y motivación en todos los niveles jerárquicos (equipo directivo, mandos intermedios y operarios) (Bessant y Caffyn, 1997; Lycke, 2000); en segundo lugar, este proceso sólo ha funcionado eficazmente impulsado por una metodología de implantación adecuada, esto es, dotando al proceso de una adecuada «estructuración»que, finalmente, es la que permite asegurar el compromiso y la motivación de toda la organización (Hanson, 200I).

Asimismo, aunque el apoyo de la dirección y de los equipos directivos es necesario (en la definición de la estrategia), la mayoría de las actividades de mejora continua se producen en los más bajos niveles de la organización (operarios y mandos intermedios). Por lo tanto, la participación del personal se convierte en una parte clave y esencial de la aplicación de la mejora continua, dado que son las personas las que sustentan buena parte de las ventajas competitivas intangibles de las organizaciones (difíciles de copiar), esto es, conocimientos, habilidades y actitudes (Teece y Pisano, 1994; Bessant et al., 1994; Terziovski y Sohal, 2000; Harman et al., 2002; García y Prado, 2002).

En este contexto, cobra importancia la implantación de sistemas estructurados de participación del personal (SPs) que, como parte fundamental de la implantación de un programa de mejora continua, ayudan a la mejora competitiva de las empresas (Laursen y Foss, 2003). Así, el Club Gestión de Calidad en 1997 (hoy denominado Club Excelencia en Gestión) definía a los sistemas de participación del personal como «actividades estructuradas de forma sistemática que permiten canalizar eficientemente todas las iniciativas que puedan incrementar la competitividad empresarial». Los sistemas estructurados de participación clásicos pueden ser tanto de tipo grupal (p.ej. círculos de calidad, grupos de mejora, etc), como individual (p.ej. sistemas de sugerencias). Todos ellos permiten una intervención activa y sistemática de los diferentes niveles jerárquicos de una organización en los procesos de mejora y, en muchos casos, han permitidos canalizar los objetivos estratégicos de muchos enfoques de mejora como los comentados anteriormente (JIT, 6 sigma, TQM,...).

Si bien, ciertos sistemas de participación como sugerencias o círculos de calidad son ampliamente conocidos en la literatura con estos nombres, esto mismo no ocurre con los grupos de mejora, dado que con este «paraguas» se agrupan múltiples sistemas con características similares, entre los que se pueden mencionar los equipos de mejora continua, los equipos de mejora, los equipos de mejora de la calidad, los equipos kaizen o los equipos de proyectos de mejora (García y Prado, 2002; Marín-García et al.; 2008).

En la literatura suele otorgarse a los sistemas de participación grupales, una mayor capacidad, tanto para involucrar y motivar a los trabajadores en la mejora de procesos, como para contribuir a una mejora de los resultados empresariales. Más aún, de acuerdo con Miklavcic et al. (2007) y Saratxaga, (2007), el nivel más avanzado en el trabajo en grupo se caracteriza por los equipos de trabajo autogestionados. No obstante, también aparecen en la literatura menciones a que los sistemas individuales de participación o sistemas de sugerencias presentan un mejor comportamiento dado que son más fáciles de implantar y presenta un menor coste interno para las empresas.

Independientemente de la modalidad escogida (grupal o individual), los SPs requieren, tanto de una metodología adecuada (o «estructurada») para sobrevivir en el tiempo y ofrecer resultados, como del apoyo decidido por parte de la dirección de la empresa, que no debe permitir que otras prioridades los hagan caer en el olvido.Tal como afirma De Benito (1998), «este tipo de programas tienen un desarrollo inicial rápido, 'enganchan' fácilmente a las personas, que ven en ellos una oportunidad de aplicar sus conocimientos y creatividad, pero pierden impulso ya que, al no estar orientados hacia los objeti- 
vos estratégicos de la empresa, aparecen nuevas prioridades que originan una percepción de menor importancia hacia los SPs, que acaban desactivándose». Este efecto de «desencanto» progresivo con la participación, también se conoce en la literatura con nombre de efecto «luna de miel».

Buena parte de las referencias en la literatura reciente que estudian la mejora continua a través de los sistemas de participación del personal, lo hacen, o bien, desde la perspectiva del estudio de casos (preferentemente, en el ámbito empresarial indus- trial más que en el de servicios), o bien, desde el análisis de la realidad empresarial en un contexto geográfico o sectorial determinado centradas, especialmente, en el nivel de desarrollo o implantación de los sistemas de participación en las empresas (muchos de ellos en un análisis más general de implantación de sistemas de gestión de la calidad). De la revisión de todas estas aportaciones, los autores han identificado una relación de factores críticos de éxito relacionada con una satisfactoria implantación de SPs con base en un programa de mejora continua (ver tabla I, realizada a partir de

Tabla 1

Factores clave para implantar un programa de mejora continua con sistemas de participación (adaptado de García et al., 2009)

\begin{tabular}{|c|c|c|}
\hline & $\mathbf{N}^{\circ}$. & FACTOR CRÍTICO DE ÉXITO \\
\hline \multirow{5}{*}{$\begin{array}{c}\text { A) FACTORES } \\
\text { MOTIVACIONALES } \\
\text { O CULTURALES }\end{array}$} & A.l. & Apoyo y compromiso de la dirección \\
\hline & A.2. & Apoyo y compromiso de los mandos intermedios \\
\hline & A.3. & Apoyo y compromiso de los participantes en el EPS (individual o grupal) \\
\hline & A.4. & Apoyo/Compromiso de los sindicatos/comité (representantes de los trabajadores) \\
\hline & A.5. & $\begin{array}{l}\text { Comprensión interna a todos los niveles del «cambio cultural» asociado a la implantación de la me- } \\
\text { jora continua }\end{array}$ \\
\hline \multirow{9}{*}{$\begin{array}{c}\text { B) FACTORES } \\
\text { METODOLÓGICOS } \\
\text { DE DISEÑO }\end{array}$} & B.l. & Selección de una estructura organizativa apropiada para implantar el SP \\
\hline & B.2. & Adopción de unas adecuadas planificación y operativa de trabajo basada en indicadores y objetivos \\
\hline & B.3. & $\begin{array}{l}\text { Adopción de una adecuada sistemática para gestionar y realizar el seguimiento de las mejoras } \\
\text { propuestas }\end{array}$ \\
\hline & B.4. & Escoger el momento más adecuado para implantar/potenciar el SP \\
\hline & B.5. & Asesoramiento externo para implantar/mantener/mejorar un SP \\
\hline & B.6. & SPs, basados en equipos de trabajo multijerárquicos y multidepartamentales \\
\hline & B.7. & Formación de los participantes en el SPs \\
\hline & B.8. & Comunicación continua de mejoras y actividades desarrolladas en el SPs a toda la organización \\
\hline & B.9. & Adoptar un sistema de reconocimiento y/o retribución para los participantes en el SPs \\
\hline \multirow{6}{*}{$\begin{array}{c}\text { C) FACTORES } \\
\text { METODOLÓGICOS } \\
\text { OPERATIVOS }\end{array}$} & C.I. & $\begin{array}{l}\text { Participación del proponente del problema/mejora en el diseño, análisis e implantación de las accio- } \\
\text { nes asociadas }\end{array}$ \\
\hline & C.2. & Énfasis en el diseño y análisis de la mejora previamente a su implantación \\
\hline & C.3. & Marcar prioridades en la implantación de las mejoras propuestas \\
\hline & C.4. & Proporcionar recursos para respuesta ágil a problemas, preguntas o propuestas \\
\hline & C.5. & Obtención de resultados desde un inicio para fortalecer la confianza en el SP \\
\hline & C.6. & $\begin{array}{l}\text { Sistematizar las mejoras obtenidas mediante el desarrollo e implantación de procedimien- } \\
\text { tos/instrucciones de trabajo }\end{array}$ \\
\hline
\end{tabular}


las síntesis y revisiones bibliográficas realizadas por Garcia et al., 2009). En la relación de factores críticos anteriores, los autores han establecido tres grandes categorías: los factores motivacionales o culturales, los factores metodológicos de diseño y los factores metodológicos operativos.

El modelo de gestión conceptual de los SPs que los autores proponen se basaría en que con una adecuada metodología de implantación (factores críticos de éxito, tanto de diseño como operativos), se logra un incremento de la motivación en la organización facilitando, asimismo, el cambio cultural asociado a la mejora continua. Todo ello repercutiría, además, en un mayor impacto positivo sobre los resultados empresariales (los directos y los indirectos). En este artículo se hace un análisis exploratorio de este modelo.

En este contexto, la escasez de un tratamiento detallado en la literatura sobre el efecto de la estructuración de los sistemas de participación (en un ámbito de implantación de mejora continua) en los resultados económicos y sociales de las empresas (mejora de la competitividad empresarial), dota a esta investigación de un carácter novedoso, más aún cuando existe evidencia de que no todas las empresas han tenido el éxito esperado (o cuando no, han fracasado) en la implantación de sus programas de mejora continua (Marín-García et al., 2008).

\section{Objetivos y metodología}

En este contexto, esta ponencia tiene un triple objetivo; por un lado, identificar y valorar los principales motivos por los que las empresas implantan sus SPs; por otro, desde la perspectiva empresarial, identificar y valorar la importancia y dificultades asociados a los múltiples factores de éxito mencionados en la literatura a la hora de implantar los SPs. Finalmente, teniendo en cuenta estos factores, se valoran los principales resultados (directos e indirectos) que las empresas están obteniendo gracias a la implantación de SPs.

En el estudio se ha contado con la participación de 40 empresas, pertenecientes a diferentes sectores, tanto industriales como de servicios, que han demostrado su compromiso en la implantación de procesos de mejora continua a través de SPs. Se partía de la dificultad de poder definir el universo de empresas españolas con SPs implantados, al no existir un registro de este tipo de actividades y sólo tener como posible referencia, básicamente, a las empre- sas que han implantado sistemas avanzados de gestión de calidad o experiencias bajo el marco del modelo de excelencia EFQM. Por ello, se contó con la colaboración de dos prestigiosas entidades implicadas en el enfoque de nuestro proyecto: el Comité de Participación y Mejora de la Asociación Española de la Calidad (AEC) y la Asociación Española de Normalización y Certificación (AENOR) que, como entidad relacionada e interesada en la promoción de la mejora continua en el ámbito empresarial, aportó un gran apoyo a través de su registro de empresas certificadas ISO 900 I.

De la muestra, I 3 empresas se corresponden con el total de las pertenecientes al Comité de Participación y Mejora de la Asociación Española de la Calidad (AEC), y las otras 37 son empresas de diferentes sectores y ubicadas en todo el territorio nacional (de éstas, 20 pertenecientes a la Comunidad Autónoma de Galicia).

En cuanto a la actividad de estas empresas (ver figura I), el $67 \%$ de ellas pertenecen al sector industrial, y de éstas un $44 \%$ son del sector automoción. El $33 \%$ restante de las empresas (13) pertenecen al sector servicios (3 de ellas son bancos y otras 3 son empresas de transportes; el resto se reparten en educación, telecomunicaciones, etc). Finalmente, cabe señalar que mayoritariamente se trata de multinacionales.

Figura I

Caracterización de la muestra de estudio

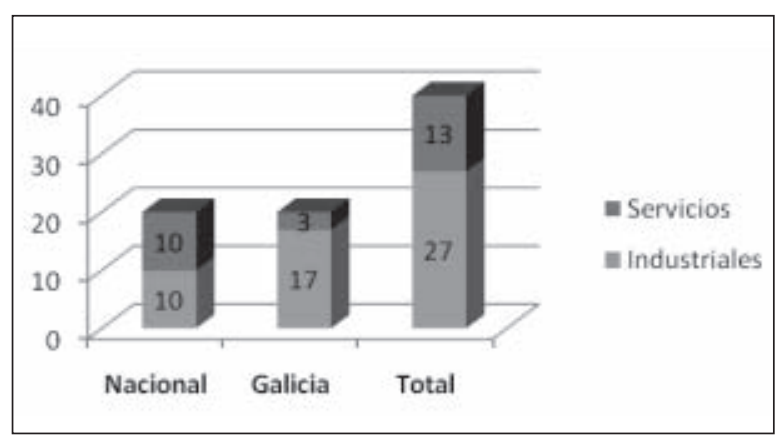

Todas las empresas cuentan con un bagaje considerable en lo que respecta a la implantación de alguna modalidad de SPs; así, el 75\% de ellas iniciaron su primer contacto con los SEP hace más de 5 años.

En cuanto a la metodología que se ha seguido para el estudio, ésta se ha fundamentado en la realización de entrevistas personales en profundidad a los responsables de los SEP de las empresas (en muchos casos la Dirección) sobre la base de un cuestionario 
de 128 preguntas, muchas de las cuales han estado orientadas con aspectos estratégicos y organizativos. De entre todas estas preguntas se han seleccionado aquellas más relacionadas con la temática de este artículo. Esta investigación ha sido posible gracias a la financiación de la Consellería de Industria de la Xunta de Galicia.

\section{Resultados}

En este epígrafe se desarrollan los resultados más relevantes del estudio, los cuales se han estructurado en tres apartados: uno primero dedicado a identificar los principales motivos por los que las empresas implantan sus SPs; a continuación le sigue un apartado donde se describe la valoración de la importancia que la empresas otorgan a cada uno de los factores de éxito (nombrados en la literatura y comentados en el epígrafe anterior) identificando, asimismo, los principales problemas con los que las empresas se encuentran al implantar estos factores críticos. Finalmente, se comentan los principales resultados (directos e indirectos) que las empresas consideran que están obteniendo con la implantación de sus SPs.

Como caracterización general de estos sistemas de participación indicar que el 63\% (25) de las empresas tienen implantados un modelo mixto de participación, es decir, que combinan sistemas grupales (grupos de mejora, círculos de calidad,...) con sistemas individuales (tipo sugerencias). En el resto de empresas, el 25\% (10) tienen implantados sólo sistemas grupales, mientras que el I 2\% (5) tienen implantados sólo sistemas individuales.

\section{I. ¿Qué motiva a las empresas a implantar sus SPs?}

Adaptando los aspectos motivadores comentados por la encuesta del CINet (CINet o Continuous Innovation Network es una organización sin ánimo de lucro que promociona el desarrollo del conocimiento en el ámbito de la mejora continua y la innovación; www.continuous-innovation.net), en el estudio se les ha preguntado a las empresas sobre los principales causas que influyeron en el lanzamiento de sus programas de SPs.

Así, se han establecidos dos tipos de aspectos: por un lado, los directamente relacionados con los resultados empresariales y por extensión claramente representados en las estrategias competitivas de las empresas (esto es, la mejora de la productividad, la reducción de los costes, la mejora del nivel de calidad, el favorecer la innovación en productos y procesos, el aumento de la flexibilidad ante cambios en el entorno, la reducción de los plazos de entrega y la mejora en el cumplimiento de los plazos de entrega) y, por otro, los indirectamente relacionados con los resultados empresariales, los cuales no siempre se reflejan nítidamente en las estrategias de las empresas al estar implícitamente relacionados con el concepto difuso de «cambio cultural» en las organizaciones; así, entre estos resultados «indirectos» se han contemplado, el servir como herramienta para la implantación de otros enfoques de mejora (ISO, Lean/IIT, EFQM, 6 sigma,... ), la mejora del «clima laboral» de la empresa, la mejora en la seguridad y las condiciones de trabajo, el aumento en el nivel formativo de los trabajadores, la posibilidad de descubrir/desarrollar nuevas capacidades entre los participantes en los SPs (liderazgo, toma de decisiones, capacidad de análisis,...), la mejora en el compromiso y la actitud de los empleados hacia el cambio, la disminución del absentismo, la mejora de la cooperación y la comunicación interna, la mejora de la satisfacción del cliente $y$, finalmente, la mejora de las relaciones entre departamentos o con proveedores y clientes.

En las tablas 2 y 3 se muestran las valoraciones (escala Likert I-5) que las empresas han otorgado a los factores señalados anteriormente a la hora de motivar la implantación de los SPs. Así, entre los factores relacionados directamente con los resultados empresariales (tabla 2), el más valorado y de forma destacada ha sido la mejora del nivel de calidad, seguido a cierta distancia de la reducción de costes y el aumento de la productividad. Este primer grupo de motivadores «directos» es significativamente más importante que el resto de motivadores «directos» considerados.

En un nivel intermedio se ubican aspectos como la contribución a la innovación de productos y servicios y el aumento de la flexibilidad ante cambios. Los aspectos menos importantes como desencadenantes del lanzamiento de un SPs son los relacionados con el plazo de entrega, tanto a nivel de su reducción como de la mejora de su nivel de cumplimiento.

En cuanto a los factores relacionados indirectamente con los resultados empresariales, se observa una valoración de la importancia bastante similar entre buena parte de los factores, salvo los asociados a la mejora de las relaciones con los proveedores y a la reducción del absentismo (con una valoración signi- 
Tabla 2

Principales motivos o desencadenantes en las empresas para implantar sus SPs en relación con los resultados directos empresariales (en negrilla principales motivos)

\begin{tabular}{|c|c|c|c|}
\hline Tipo de motivo & Promedio & Varianza & Estadístico \\
\hline Mejorar la calidad & 4,158 & 0,623 & \multirow{7}{*}{$\begin{array}{c}\text { ANOVA } \\
\mathrm{F}=7,50284 ; \\
\text { p-value }= \\
2,06739 \mathrm{E}-07<0,05\end{array}$} \\
\hline Reducir los costes & 3,650 & 1,310 & \\
\hline Mejorar la productividad & 3,553 & $\mathrm{I}, \mathrm{I} 73$ & \\
\hline Favorecer la innovación en productos y servicios & 3,205 & ।,904 & \\
\hline Mejorar la flexibilidad de la organización & 3,053 & 1,619 & \\
\hline Reducir los plazos & 2,758 & 1,564 & \\
\hline Mejorar el cumplimiento de plazos (fiabilidad) & 2,606 & 1,746 & \\
\hline
\end{tabular}

Tabla 3

Principales motivos o desencadenantes en las empresas para implantar sus SPs en relación con los resultados indirectos empresariales (en negrilla los principales motivos)

\begin{tabular}{|c|c|c|c|}
\hline Tipo de motivo & Promedio & Varianza & Estadístico \\
\hline Mejorar la comunicación interna & 3,763 & 0,888 & \multirow{12}{*}{$\begin{array}{c}\text { ANOVA } \\
\text { F=9, I } 4 \text { I } 89 ; \\
\text { p-value }= \\
8,362024 \mathrm{E}-15<0,05\end{array}$} \\
\hline Mejorar la seguridad y en las condiciones de trabajo & 3,667 & 1,544 & \\
\hline Mejorar la satisfacción de los clientes & 3,568 & $1,64 \mid$ & \\
\hline $\begin{array}{l}\text { Servir de base para implantar otras enfoques de mejora (Lean, JIT, } 6 \text { sig- } \\
\text { ma,...) }\end{array}$ & 3,526 & ।,932 & \\
\hline Mejorar la actitud de la organización hacia el cambio & 3,500 & 1,014 & \\
\hline Mejorar las relaciones entre departamentos & 3,500 & 1,230 & \\
\hline Mejorar el «clima» laboral interno & 3,308 & 1,482 & \\
\hline Mejorar las capacidades y la formación de las personas & 3,282 & 1,260 & \\
\hline Descubrir nuevas capacidades entre los participantes en los SPs & 3,184 & ।,235 & \\
\hline Mejorar las relaciones con los clientes & 2,947 & ।,457 & \\
\hline Mejorar las relaciones con los proveedores & 2,444 & 2,025 & \\
\hline Reducir el absentismo & ।,730 & ।,036 & \\
\hline
\end{tabular}

ficativamente menor). Destaca como primera motivación la mejora de la cooperación y la comunicación, seguido a corta distancia, tanto de la mejora de la seguridad y las condiciones de trabajo, la mejora de la satisfacción del cliente y el servir de base para implantar otros enfoques de mejora, como de una mejor actitud hacia el cambio o la mejora en las relaciones entre departamentos.
En una posición intermedia se encuentran, la mejora del «clima» interno, la mejora de las capacidades y la formación de las personas, el descubrimiento de nuevas capacidades entre los participantes en los SPs y la mejora de las relaciones con los proveedores.

De forma cualitativa, algunas de las empresas entrevistadas (6; en particular, pertenecientes a grupos 
multinacionales) manifiestan que el «empujón» definitivo para lanzar (o potenciar) sus SPs ha sido la extensión de los modelos ya implantados en otras partes del grupo (más o menos adaptados a la realidad local.

Otros «empujones» para lanzar/promocionar los SPs son los motivados por la implantación de diferentes programas con enfoques de mejora (Lean, EFQM,..) (2 empresas), por las recomendaciones de un equipo consultor externo (2 empresas), la incorporación de empresas de un nuevo gerente/director/responsable que ya ha conocido en experiencias profesionales anteriores el potencial de mejora vinculado a la implantación de sistemas de participación ( I empresa) o el convencimiento del potencial de los SPs a partir de visitas realizadas a empresas externos (en I empresa).

\subsection{Factores críticos en la implantación de los SPs: importancia y problemas asociados}

Para poder evaluar y ordenar la importancia de cada uno de los 20 factores críticos comentados en la tabla I, se ha pedido a las empresas analizadas que los valoren en la escala Likert ( I -5; ver tabla 4), tanto los asociados a los aspectos motivacionales como los vinculados a los aspectos metodológicos (de diseño y operativos).

Del análisis de estas opiniones vertidas por las empresas se extrae que existen diferencias significativas entre las valoraciones de cada factor (ver tabla 4). Así, los tres factores que presentan una puntuación por encima del 4,5 de media en la valoración y que significativamente las empresas analizadas conside-

Tabla 4

Factores críticos de éxito en la implantación de los SPs: importancia y dificultades (entre paréntesis las principales posiciones del ranking en importancia y dificultades, respectivamente)

\begin{tabular}{|c|c|c|c|c|c|c|c|}
\hline & & \multicolumn{3}{|c|}{ IMPORTANCIA } & \multicolumn{3}{|c|}{ DIFICULTADES } \\
\hline & & Promedio & Varianza & Estadístico & Promedio & Varianza & Estadístico \\
\hline \multirow{5}{*}{$\begin{array}{l}\text { A) FACTORES } \\
\text { MOTIVACIONALES } \\
\text { O CULTURALES }\end{array}$} & A.l. & $4,769(I)$ & 0,551 & \multirow{20}{*}{$\begin{array}{c}\text { ANOVA } \\
\text { F=9,6894; } \\
\text { P-value= } \\
\text { I,3865E-25 } \\
<0,05\end{array}$} & 1,838 & 1,640 & \multirow{20}{*}{$\begin{array}{l}\text { ANOVA } \\
\begin{array}{c}\text { F = 4,6055; } \\
\text { P-value }= \\
4,673 \mathrm{E}-10 \\
<0,05\end{array}\end{array}$} \\
\hline & A.3. & $4,692(2)$ & 0,271 & & $2,324(7)$ & 1,114 & \\
\hline & A.2. & $4,564(3)$ & 0,410 & & $2,622(1)$ & 1,242 & \\
\hline & A.5. & $4,056(10)$ & 1,311 & & $2,529(4)$ & 1,045 & \\
\hline & A.4. & 2,784 & 2,619 & & 1,939 & 2,621 & \\
\hline \multirow{9}{*}{$\begin{array}{l}\text { B) FACTORES } \\
\text { METODOLÓGICOS } \\
\text { DE DISEÑO }\end{array}$} & B.3. & $4,132(7)$ & 1,036 & & $2,189(9)$ & 1,102 & \\
\hline & B.8. & 4,077 (9) & 0,704 & & 2,027 & $0,97 \mid$ & \\
\hline & B.4. & $4,053(\mathrm{II})$ & 0,862 & & $2,429(5)$ & 1,664 & \\
\hline & B.I. & $4,051(12)$ & 1,366 & & 2,081 & 0,910 & \\
\hline & B.2. & $4,026(13)$ & 1,236 & & $2,270(8)$ & 1,203 & \\
\hline & B.9. & 3,923 & 1,336 & & 1,919 & 1,299 & \\
\hline & B.6. & 3,897 & 1,305 & & 1,457 & 0,726 & \\
\hline & B.7. & 3,641 & 1,868 & & $|, 54|$ & 0,700 & \\
\hline & B.5. & 2,500 & 1,446 & & 1,206 & 0,229 & \\
\hline \multirow{6}{*}{$\begin{array}{l}\text { C) FACTORES } \\
\text { METODOLÓGICOS } \\
\text { OPERATIVOS }\end{array}$} & C.6. & $4,205(4)$ & 0,852 & & $2,568(3)$ & 1,530 & \\
\hline & C.5. & $4,205(5)$ & 1,167 & & 1,944 & 0,911 & \\
\hline & C.2. & $4,154(6)$ & 1,344 & & 2,054 & 1,053 & \\
\hline & C.4. & $4,128(8)$ & 0,746 & & $2,622(2)$ & $1,13 \mid$ & \\
\hline & C.3. & 3,949 & 0,839 & & $2,35 I(6)$ & 1,345 & \\
\hline & C.I. & 3,947 & 1,240 & & 2,111 & 1,130 & \\
\hline
\end{tabular}


ran como más importantes son: «El compromiso de la Dirección», «El compromiso y motivación de los participantes» y «El compromiso de los mandos intermedios». Por tanto, las empresas otorgan la mayor criticidad para implantar exitosamente sus SPs a los aspectos de motivación y compromiso de toda la organización (en todos los niveles jerárquicos); entre estos factores, la literatura menciona más profusamente la importancia de la involucración de la dirección.

En un segundo nivel de importancia (estadísticamente) se encuentran un conjunto de 14 factores entre los que no se encuentran diferencias significativas. Entre estos factores, representativos de los tres grupos considerados (motivacionales, de diseño y operativos) se encuentran algunos especialmente mencionados en la literatura como la participación del personal en el diseño e implantación de las mejoras, el cambio cultural vinculado a la mejora continua, el trabajo en equipo, la disponibilidad de recursos, la comunicación, la estandarización y documentación de procesos o los sistemas de incentivos/recompensa.

A otro menor nivel de importancia (estadísticamente) se encuentran la formación de los participantes en el funcionamiento de los SPs; la relativa poca importancia otorgada por las empresas a este aspecto no deja de ser curiosa, teniendo en cuenta la abundancia de referencias a esta materia encontradas en la literatura. Finalmente, en el último grupo de factores aparecen, como los menos críticos o importantes, el apoyo de los sindicatos y el asesoramiento externo.

Por otro lado, si se analiza en qué factores críticos las empresas han encontrado más problemas al implantar sus SPs, se concluye que los factores más complicados (significativamente) son: el compromiso de los mandos intermedios, la disponibilidad de recursos para poder abordar las mejoras analizadas, la sistematización o estandarización de las mejoras alcanzadas, la comprensión del cambio cultural asociado a la mejora continua, la selección del momento más adecuado para el lanzamiento del SPs, la selección del mejor momento para implantar las mejoras, la obtención de un mayor compromiso entre los participantes en el SPs y el uso de una metodología de implantación basada en indicadores y objetivos.

Por el contrario, los aspectos metodológicos con los que las empresas se han encontrado menos problemas a la hora de implantar sus SPs son: el compro- miso de la dirección (el aspecto más valorado por las empresas en cuanto a importancia), la formación a los participantes, la constitución de equipos de trabajo multijerárquicos y multidepartamentales (en las empresas que tienen SPs de carácter grupal) y el asesoramiento externo (coincide con el aspecto menos importante desde el punto de vista de las empresas).

\section{3. ¿Qué están obteniendo las empresas al implantar sus SPs?}

Finalmente, en esta sección del apartado de resultados se presentan las valoraciones que las empresas hacen de la contribución de los SPs (una vez implantados) a la mejora de los resultados empresariales (los directos y los indirectos).

Así, las empresas destacan el impacto positivo de los SPs en los resultados directos (ver tabla 5), relativos a la mejora de la calidad, la reducción de los costes y la mejora de productividad (aspectos que eran mayoritariamente, los motivos principales para implantar los SPs). En el otro extremo, los resultados más pobres alcanzados por las empresas con la implantación de sus SPs, tienen que ver con la mejora de la flexibilidad, la mejora de los plazos de servicio y de la fiabilidad de estos plazos.

No obstante, es importante señalar que no se han encontrado diferencias estadísticamente significativas en la valoración de las expectativas iniciales y la valoración del impacto en los resultados directos. La excepción a este último comentario viene dado por las diferencias encontradas en los aspectos de «calidad» e «innovación», donde la valoración de los resultados alcanzados son significativamente menores que las expectativas iniciales (concretamente, en calidad el Estadístico $\mathrm{t}=3,207$ y $\mathrm{P}(\mathrm{T}<=\mathrm{t})=0,00198$; en innovación el Estadístico $t=2,6189$ y $\mathrm{P}(T<=\mathrm{t})=$ 0,0 10836).

Por otro lado, en cuanto a la contribución de los SPs a la mejora de los resultados indirectos (ver tabla 6), indicar que las empresas señalan mayoritariamente, la mejora de las relaciones entre departamentos (que no era el principal motivo para su implantación) seguido por la mejora del compromiso de la organización hacia el cambio (tampoco se encontraban entre las principales expectativas) y la mejora de la cooperación y la comunicación entre personas. Desde la perspectiva de contribución a los resultados indirectos, las peores valoraciones tienen que ver, tanto con la mejora de las relaciones con clientes y proveedores, como con la reducción de la tasa de 
Tabla 5

Valoración de la contribución de los SPs a la mejora de los resultados directos de las empresas (se incluye el promedio de la valoración de los motivos iniciales para facilitar la comparativa)

\begin{tabular}{|c|c|c|c|c|}
\hline \multirow[b]{2}{*}{ Contribución a resultados } & \multirow{2}{*}{$\begin{array}{l}\text { MOTIVOS } \\
\text { Promedio }\end{array}$} & \multicolumn{3}{|c|}{ RESULTADOS } \\
\hline & & Promedio & Varianza & Estadístico \\
\hline CALIDAD & 4,158 & 3,526 & 0,851 & \multirow{7}{*}{$\begin{array}{c}\text { ANOVA } \\
\text { F=7,738232; } \\
\text { p-value }= \\
\text { 1,2297E-07 } \\
<0,05\end{array}$} \\
\hline COSTES & 3,650 & 3,474 & 1,175 & \\
\hline PRODUCTIVIDAD & 3,553 & 3,421 & 1,385 & \\
\hline FLEXIBILIDAD & 3,053 & 2,816 & 1,289 & \\
\hline REDUCIR PLAZOS & 2,758 & 2,600 & 1,659 & \\
\hline INNOVACIÓN & 3,205 & 2,375 & 1,597 & \\
\hline CUMPLIR PLAZOS & 2,606 & 2,314 & 1,339 & \\
\hline
\end{tabular}

Tabla 6

Valoración de la contribución de los SPs a la mejora de los resultados indirectos de las empresas (se incluye el promedio de la valoración de los motivos iniciales para facilitar la comparativa)

\begin{tabular}{|c|c|c|c|c|}
\hline \multirow[b]{2}{*}{ Contribución a resultados } & \multirow{2}{*}{$\begin{array}{l}\text { MOTIVOS } \\
\text { Promedio }\end{array}$} & \multicolumn{3}{|c|}{ RESULTADOS } \\
\hline & & Promedio & Varianza & Estadístico \\
\hline RELACIONES DEPARTAMENTOS & 3,500 & 3,632 & 1,050 & \multirow{12}{*}{$\begin{array}{c}\text { ANOVA } \\
\mathrm{F}=10,04242 ; \\
\mathrm{p}-\mathrm{value}= \\
2,22098 \mathrm{E}-16 \\
<0,05\end{array}$} \\
\hline MEJORAR ACTITUD HACIA EL CAMBIO & 3,500 & 3,605 & 1,543 & \\
\hline COMUNICACIÓN & 3,763 & 3,553 & 0,903 & \\
\hline BASE PARA OTROS ENFOQUES DE MEJORA & 3,526 & 3,432 & 2,197 & \\
\hline SEGURIDADY CONDICIONES DETRABAJO & 3,667 & 3,297 & 1,215 & \\
\hline SATISFACCIÓN CLIENTES & 3,568 & 3,263 & 1,605 & \\
\hline CAPACIDADESY FORMACIÓN & 3,282 & 3,237 & 1,159 & \\
\hline CLIMA LABORAL & 3,308 & 3,237 & 0,834 & \\
\hline NUEVAS CAPACIDADES ENTRE INTEGRANTES & 3,184 & 3,105 & 1,286 & \\
\hline RELACIONES CLIENTES & 2,947 & 2,763 & 2,240 & \\
\hline RELACIONES PROVEEDORES & 2,444 & 2,306 & 2,104 & \\
\hline ABSENTISMO & 1,730 & $|, 54|$ & 0,533 & \\
\hline
\end{tabular}

absentismo (tanto en expectativas iniciales como en resultados alcanzados).

Asimismo, en el análisis comparativo entre los diferentes aspectos vinculados a los resultados indirectos y sus expectativas iniciales se concluye que no existen diferencias estadísticamente significativas.

Finalmente, indicar que en el 75\% de las empresas la estimación de estos resultados procede de la propia valoración subjetiva del entrevistado, careciendo de base cuantitativa para validar la puntuación, lo que también matizaría la interpretación de los comentarios desarrollados en este epígrafe.

\section{Algunas reflexiones}

De los resultados del estudio precedente se puede concluir que las empresas han adoptado SPs grupales o individuales más allá de los esquemas tradicionales y lo han hecho adaptando intuitivamente buena parte de los factores críticos anteriormente comentados. No obstante, el éxito de esta implanta- 
ción ha sido dispar, si bien, en general bastante satisfactorio en base a la propia valoración global de las empresas (valor de 3,75 en una escala I-5 de Likert).

No obstante, ¿en qué pueden mejorar metodológicamente las empresas para incrementar el impacto en los resultados empresariales, directos e indirectos? Las empresas destacan la importancia para lograr la motivación de la organización, tanto a nivel de trabajadores y mandos intermedios, como del equipo directivo. En este sentido, las empresas analizadas están más satisfechas con la indispensable involucración compromiso de la dirección y el equipo directivo que con los mandos intermedios y los propios operarios. Especialmente relevante es la dificultad encontrada para involucrar a los mandos intermedios (el «eslabón débil» a juicio de muchos entrevistados), teniendo en cuenta la importancia que este factor tiene para la implantación de la participación y como correa de transmisión de la estrategia empresarial vinculado al cambio cultural asociado a la mejora continua.

Asimismo, entre los aspectos motivacionales sorprende la poca importancia otorgada a los representantes de los trabajadores (sindicatos), si bien, tampoco existe una oposición frontal de éstos a la implantación de los SPs, tal como se ha visto a la hora de valorar los problemas acaecidos con la implantación de los SPs. No obstante, es un motivo de reflexión, la necesidad de una mayor proactividad de los representantes sindicales en un contexto de cambio de modelo de relaciones más allá del conflicto entre la «patronal»y los «trabajadores» (de nuevo, el «cambio cultural»).

Sin embargo, parece que el problema con esta involucración no está directamente vinculado a la organización adoptada para implantar los SPs, dado que el $70 \%$ de las empresas analizadas afirman disponer de una estructura organizativa definida (con niveles jerárquicos y responsabilidades asignadas) para la gestión de los SPs implantados y algunas más, un $80 \%$ de las empresas, cuentan con una o varias personas que actúan como responsbles/coordinadores/animadores de las actividades de participación (evidentemente, no necesariamente, a tiempo completo).

Si el problema no está directamente relacionado con la estructura adoptada, sí parece que puede estar relacionado con la falta de un seguimiento sistemático de las actividades de los SPs basado en indicadores y objetivos de gestión. Esta falta de indicadores y objetivos, precisamente, puede ser una de las causas que impida vincular de forma más directa los resul- tados empresariales (directos e indirectos) con las actividades adoptadas por los SPs.

En este contexto, si bien el $85 \%$ de las empresas disponen de algún tipo de indicador asociado a las actividades de los SPs, sólo un 33\% manifiesta disponer de un sistema detallado de los mismos. En esta línea, sólo el $51 \%$ define objetivos referentes a la propia actividad de los SPs (p.e.\% plantilla que se desea que participe, $\%$ de acciones de mejora implantadas a alcanzar en un período de tiempo...).

En otro orden de cosas, en relación con el sistema de retribución o reconocimiento (desde el punto de vista de los autores fundamental el reconocimiento por participar no vinculado directamente a una recompensa económica) el análisis hay que realizarlo por tipo de modalidad de SP adoptada. Así entre las 35 empresas analizadas que disponen de SPs grupales el $49 \%$ no considera oportuno llevar a cabo ningún tipo de reconocimiento/recompensa, mientras que entre las 30 empresas con sistemas individuales el 70\% realiza algún tipo de recompensa o reconocimiento por la participación (si bien, un 57\% de éstas lo hacen mediante una recompensa económica).

Por otro lado, a nivel de la comunicación y la difusión interna de las actividades llevadas a cabo en los SPs, aunque hay cierta diversidad respecto a cómo se realiza la comunicación y a quiénes va dirigida, el 72\% de las empresas analizadas afirman que siempre realizan algún tipo de comunicación a toda la organización al inicio o lanzamiento de una nueva modalidad de SP (o modificación de uno existente), mientras que un $11 \%$ manifiesta no realizar ninguna comunicación especial en este lanzamiento o promoción de los SPs. La valoración media otorgada por las empresas respecto a la realización de esta comunicación inicial es de un 4,3 (en una escala 1-5, donde I significa que no la realizan nunca, y 5 que la realizan siempre).

Sin embargo, cuando se trata de informar sistemáticamente a toda la organización de los resultados/acciones que van emanando de los SPs, este porcentaje baja hasta el $42 \%$ que lo hace siempre, por un $25 \%$ que manifiesta que no lo realiza nunca. La valoración media en este caso cae hasta el 3,43. Esta comunicación sí está más sistematizada cuando se trata de informar de los resultados anuales del programa de SPs, o tras el cierre de cada una de las experiencias grupales: el 66\% manifiesta hacerlo siempre, por un 13\% que nunca lo hace. La valoración media es de 4,08, superior a la comunicación duran- 
te el proceso, pero ligeramente por debajo de la comunicación inicial.

Por último, profundizando en los comentarios anteriores también se ha analizado hasta qué punto la modalidad de SPs implantado (grupal, individual o mixto) pueden afectar positiva o negativamente a una mejor posición competitiva, esto es, a mejorar los resultados empresariales (directos e indirectos). Así, indicar que se han encontrado diferencias significativas en algunos de los resultados analizados; concretamente, se han identificado en las empresas que han adoptado SPs grupales (solos o combinados con sistemas individuales) unos mejores resultados en productividad (resultados directos; $F=4,30$ । 47; $p$-value $=0,02$ | $61 \mid<0,05)$ y mejor actitud hacia el cambio (resultados indirectos; $F=4,4349$; $P$-value $=0,0192<0,05)$.

Además, la valoración global de la importancia de los SPs para contribuir a la competitividad de las empresas es mayor en las empresas con sistemas grupales $(F=8,00226$; $p$-value $=0,001374<0,05)$. Los comentarios anteriores parecen validar las aportaciones de la literatura que plantean más ventajas y mejores resultados en los sistemas grupales que en los sistemas individuales.

\section{Conclusiones}

En un mundo globalizado, caracterizado por unos altos niveles de competencia que afectan a todos los sectores, independientemente de su carácter industrial o de servicios, las empresas deben emprender acciones que les permitan no sólo mantener, sino deseablemente, mejorar su posición competitiva. En este contexto, las empresas plantean diferentes actuaciones vinculadas a su estrategia, entre las que se puede encontrar la adopción de un programa de mejora continua mediante el diseño e implantación de un programa de SPs.

De cualquier forma, hoy en día, casi nadie discute el interés y la contribución de los SPs a la mejora de la posición competitiva de las empresas, lo que se puede ilustrar por la importancia que las empresas analizadas les otorgan y por su contribución activa a la mejora de los resultados empresariales (directos e indirectos). Sin embargo, los motivos, desencadenantes o expectativas que impulsan a las empresas a lanzarse a este tipo de programas presentan diferentes matices tal como se ha comentado a lo largo de este artículo.
En esta misma línea, buena parte de las aportaciones de la literatura reciente en relación con la implantación de la mejora continua y, más concretamente, con la implantación de SPs destacan la necesidad estructurar esta implantación, lo que está directamente relacionado con la adopción de diversos factores críticos de éxito (ver tabla I). En este artículo, desde la perspectiva de las empresas, se han ordenado estos factores críticos (los principales los de carácter organizativo) identificándose y comentándose, asimismo, los principales problemas encontrados con la metodología de implantación (por ejemplo, el uso de indicadores y objetivos, el diseño de un sistema de recompensa, o la definición de una sistemática de comunicación y difusión de las actividades de los SPs).

Es importante resaltar que la implantación de SPs en las empresas está evolucionando en ocasiones desde las modalidades clásicas hacia versiones más adaptadas a las necesidades de cada organización (por ejemplo, sistemas de sugerencias más en línea con ideas de progreso o iniciativas de mejora y sistemas grupales más tipo workshops, subcomités o incluso, agrupaciones particulares bajo metodología Lean Manufacturing o Seis Sigma). No obstante, de manera práctica, la filosofía y el funcionamiento de estos medios de participación siguen siendo similares.

Por último, señalar que la aportación de los SPs a la mejora competitiva y, en particular, a cómo adoptar una adecuada metodología de implantación (que se ha iniciado en este artículo) será objeto de futuras investigaciones por parte de los autores. Como limitaciones del estudio planteado, indicar el reducido número de empresas analizado (40), así como el carácter subjetivo de las respuestas cuantitativas planteadas a las empresas que podrían matizar algunas de las observaciones comentadas en el mismo.

\section{Referencias}

BESSANT, J., CAFFYN, S.Y GILBERT, J. ( 1994), «Mobilising continuous improvement for strategic advantage» in Platts, K.W., Gregorj', M.J. and Neely, A. (Eds), Operations Strategy and Performance, Manufacturing Engineering Group, University' of Cambridge, Cambridge, pp. 175-80.

BESSANT, J.Y CAFFYN, S. (1997), «High involvement innovation through continuous improvemenf», Intemational Joumal of Technology Management, Vol. I 4 No. I, p. 7-28.

BOER, H., BERGER, A., CHAPMAN, R. Y GERTSEN, F. (eds.) $(2000) \ll \mathrm{Cl}$ changes. From suggestion box to organisa- 
tional learning. Continuous improvement in Europe and Australia», Ashgate, Aldershot.

BOER, H. (200I) «And [lethro] said ... Learning: the link between strategy, innovation and production», Center for Industrial Production, Aalborg University, 4 May.

BOLWIJN, P.T.Y KUMPE, T. (1998) «Marktregicht ondernemen. Management van continuïteit en vernieuwing», Van Gorcum, Assen.

DE BENITO, C.M. (1998): «Guía para la implantación de la calidad total en pymes», Alta Dirección, No. 197, pp. 13-27.

GARCÍA, J.y PRADO, J.C. (2002) «La participación del personal en la mejora continua de las empresas», Edited by AEC, Madrid, Spain.

GARCÍA, J., MEJIAS, A., FERNÁNDEZ, A. y PRADO, J.C. (2009), «El desarrollo de la mejora continua a través de Sistemas Estructurados de Participación del Personal (SEPP). Propuesta de una metodología de implantación», Libro de Ponencias. $3^{\circ}$ Congreso Internacional de Ingeniería de Organización 2009 (Barcelona).

GRÜTTER, A.W., FIELD, J.M.Y FAULL, N.H.B. (2002) «Work team performance over time: three case studies of South African manufacturers», Journal of Operations Management, Vol. 20, pp. 64 I-657.

HANSON, J. (200I) Implementation ofTQM, in small organizations, experiences from award winners in Sweden. Licentiate Thesis 200 I: I 3, Luleå University of Technology, Luleå.

HARMAN, R.A., GOLHAR, D.Y. y DESHPANDE, S.P. (2002) «Lessons learnt in work teams», Product Planning \& Control,Vol. 13, pp. 362-369.
JORGENSEN, F.; LAUGEN, B.Y BOER, H. (2006) «Human Resource Management for Continuous Improvement», CINet 2006, pp. 443-454.

LAURSEN, K.Y FOSS, N.J. (2003) «New human resource management practices, complementarities, and the impact on innovation performance», Cambridge Journal of Economics, vol. 27, no. 2, pp. 243-263.

LYCKE, L. (2000) «Implementing total productive maintenance, driving forces and obstacles», Licentiate Thesis 2000: 15, Luleå University of Technology, Luleå.

MARÍN-GARCÍA, J.A., PARDO DEL VAL, M. y BONAVÍA MARTÍN,T. (2008) «Longitudinal study of the results of continuous improvement in a company», Team Performance Management, Vol. I 4, pp. 55-69.

MIKLAVCIC, M., KOLENC, I., MARKIC, M. (2007), «Teamwork and defining group structures», Team Performance Management, Vol. 13 Issue 3, pp. I02- I 16.

MODARRESS, B., ANSARI, A. y LOCKWOOD, D.L. (2005), «Kaizen costing for lean manufacturing: a case study», International Journal of Production Research, Vol. 43 No. 9, pp. |75|-60.

RAPP, C. Y EKLUND, J. (2002), «Sustainable development of improvement activities: the long-term operation of a suggestion scheme», Total Quality Management, Vol. 13 No. 7, pp- 945-69.

SARATXAGA, K. (2007), «Un Nuevo estilo de relaciones para el cambio organizacional pendiente», edited by Prentice Hall (Madrid).

TERZIOVSKI, M.Y SOHAL, A.S. (2000), «The adoption of continuous improvement and innovation strategies in Australian manufacturing firms», Technovation, Vol. 20 No. 10, pp. 539-50. 